\title{
Medicamentos causantes de hepatotoxicidad en el embarazo: revisión estructurada
}

\section{A Structured Review of Hepatotoxic Medicines during Pregnancy}

Alejandra Cano P., ${ }^{1}$ Pedro Amariles, PhD. ${ }^{2}$

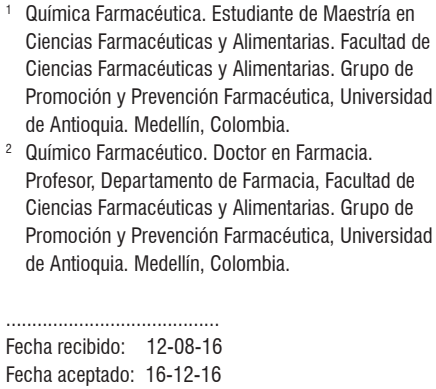

\begin{abstract}
Resumen
Objetivo: identificar los medicamentos y determinar las principales características asociadas con hepatotoxicidad por medicamentos en el embarazo. Método: revisión estructurada en PubMed/Medline, EMBASE y Web of Science utilizando los términos: Drug induced liver injury OR Hepatotoxicity AND Pregnancy. La búsqueda incluyó artículos en inglés, español, humanos, entre 2005 y 2015, con información sobre hepatotoxicidad por medicamentos en el embarazo. Fueron excluidos los artículos sin relación con embarazo o hepatotoxicidad, asociados con otras causas de enfermedad hepática o con hepatotoxicidad por otras sustancias. La información del medicamento y las características de los pacientes fueron registradas en una tabla. La probabilidad de la aparición de hepatotoxicidad fue valorada y agrupada en tres categorías: definida, probable y posible; para algunos medicamentos fue determinada por el método RUCAM. Resultados: fueron identificados 488 artículos, de los cuales 46 fueron seleccionados. Fueron identificados también 12 medicamentos (acetaminofeno, alfametildopa, labetalol, metotrexato, saquinavir, nevirapina, propiltiouracilo, metimazol, carbimazol, nitrofurantoína, ácido acetilsalicílico y piperidolato) con probabilidad de causar hepatotoxicidad en el embarazo. Algunas características asociadas con los fármacos fueron: tiempo de aparición de las reacciones, semanas de embarazo (3-36), factores de riesgo (edad y enfermedades crónicas), manifestaciones clínicas (elevación de transaminasas, prurito, ictericia) y desenlaces (trasplante de hígado, muertes materna y fetal). Conclusión: los medicamentos acetaminofeno, alfametildopa, labetalol, metotrexato, saquinavir, nevirapina, propiltiouracilo, metimazol, carbimazol, nitrofurantoína, ácido acetilsalicílico y piperidolato podrían causar hepatotoxicidad en pacientes embarazadas. Además de la dosis y del tiempo de exposición al fármaco, la edad y el tiempo de gestación podrían influir en la presentación y gravedad de la hepatotoxicidad.
\end{abstract}

Palabras clave

Hepatotoxicidad, embarazo, enfermedad hepática inducida por medicamentos.

\section{INTRODUCCIÓN}

La prescripción de medicamentos en el embarazo requiere de conocimientos adicionales, en especial, de los cambios fisiológicos que puedan generar variabilidad farmacocinética y farmacodinámica, al igual que la posible toxicidad fetal de los medicamentos (1). Relacionado con este último aspecto, se recomienda que, al momento de la prescripción, se consideren las categorías de riesgo de los medicamentos para el feto:
- A: no hay riesgos fetales en ningún trimestre.

- B: no se han demostrado riesgos fetales en estudios sobre animales.

- C: estudios en animales demuestran efectos adversos en el feto.

- D: hay una evidencia clara de riesgo fetal usados bajo riesgo-beneficio

- X: contraindicados en el embarazo por evidencia definida de daño fetal (2-4). 


\begin{abstract}
Objective: The aim of this study is to identify hepatotoxic drugs and determine the main characteristics of drug hepatotoxicity in pregnancy. Method: We conducted a structured review of PubMed/Medline, EMBASE and Web of Science using the terms "Drug induced liver injury" OR Hepatotoxicity AND Pregnancy. The search included articles in English and Spanish with information on hepatotoxic drugs in pregnancy that were posted between 2005 and 2015. Items not related to pregnancy or hepatotoxicity or which were related to other causes of liver disease or to hepatotoxicity due to other substances were excluded. Drug information and patient characteristics were recorded in a table. The probability of occurrence of hepatotoxicity was assessed and grouped into three categories: defined, probable, and possible. The categories of some drugs were determined with the RUCAM method. Results: We identified 488 articles of which 46 were selected. Twelve agents that are potentially hepatotoxic for pregnant women were identified: acetaminophen, alphamethyldopa, methotrexate, methotrexate, saquinavir, nevirapine, propylthiouracil, methimazole, carbimazole, nitrofurantoin, acetylsalicylic acid and piperidolate. Some characteristics associated with the drugs were time of reaction onset, weeks of pregnancy (3-36), risk factors (age and chronic diseases), clinical manifestations (elevation of transaminases, pruritus, and jaundice) and outcomes (liver transplantation and death of mother and/or fetus). Conclusion: Acetaminophen, alpha-methyldopa, labetalol, methotrexate, saquinavir, nevirapine, propylthiouracil, methimazole, carbimazole, nitrofurantoin, acetylsalicylic acid and piperidolate can cause hepatotoxicity in pregnant patients. In addition to dosage and exposure time, patient age and gestation time can influence presentation and severity of hepatotoxicity.
\end{abstract}

\title{
Keywords
}

Hepatotoxicity, pregnancy, drug-induced liver disease.

En este contexto, además de la teratogénesis, la toxicidad hepática es un riesgo significativo para el feto e incluso para la gestante $(1,4)$.

En el embarazo pueden presentarse algunas alteraciones gastrohepáticas propias de esta condición, las cuales se revierten después del parto. Entre ellas se destacan la hiperémesis gravídica, la colestasis gravídica, la toxemia gravídica, el síndrome HELLP y la esteatosis aguda del embarazo, que se acompañan de síntomas inespecíficos, tales como náuseas, vómitos, ictericia, prurito y aumento de las transaminasas (5). En este contexto, es necesario que el médico identifique estas alteraciones y las diferencie de posibles lesiones hepáticas asociadas con la utilización de fármacos. Esta labor puede favorecerse con la disponibilidad de información precisa y confiable de los medicamentos y demás sustancias que puedan causar toxicidad hepática para la madre y/o daños en el feto.

Aunque se dispone de información sobre la toxicidad hepática por medicamentos en el embarazo, tales como los macrólidos y las tetraciclinas (6), los antirretrovirales (nevirapina) (6-8), los psiquiátricos (litio, carbamazepina) (9) y los antitiroideos (propiltiouracilo y metimazol) (10, 11), dicha información es fraccionada y tiene limitaciones sobre las características que podrían favorecer la presentación de este evento adverso. En este sentido, los objetivos de este trabajo fueron identificar los medicamentos y determinar las principales características asociadas con hepatotoxicidad por medicamentos en el embarazo.

\section{MÉTODO}

\section{Revisión estructurada}

Se llevó a cabo una revisión estructurada en PubMed/ Medline, EMBASE y Web of Science utilizando los términos: Drug induced liver injury OR Hepatotoxicity AND Pregnancy. En la búsqueda se filtros tales como: artículos en inglés y español, en humanos y mujeres, con acceso a texto completo y artículos con las palabras clave en el título o el resumen, entre 2005 y 2015. Se incluyeron artículos con información relacionada con hepatotoxicidad en el embarazo causada exclusivamente por medicamentos. Por su parte, se excluyeron los artículos con información sin relación con el embarazo o con hepatotoxicidad por medicamentos, al igual que los relacionados con otras causas de enfermedad hepática o por otro tipo de sustancias. Para ampliar las fuentes de información, se tuvieron en cuenta también las referencias de los artículos incluidos y considerados relevantes para el tema. Los artículos fueron revisados y la información extraída por dos revisores.

De los artículos incluidos se registró la siguiente información: grupo farmacológico, fármaco, incluida la cantidad de artículos que identifican el evento y la clasificación del riesgo en embarazo según la FDA, la dosis (mg/día) utilizada, el tiempo de aparición del evento, las manifestaciones clínicas, la probabilidad de hepatotoxicidad $(12,13)$, la prevalencia de hepatotoxicidad por este fármaco, las semanas 
de gestación, los factores de riesgo, el desenlace, la forma como se trató el evento y los valores de enzimas hepáticas.

\section{Valoración de la aparición de hepatotoxicidad}

La valoración de la aparición de hepatotoxicidad se basó en la probabilidad de su ocurrencia. Esta se estableció en tres categorías, consecuentes con el nivel de evidencia encontrado:

- Definida: evidencia en metaanálisis, revisiones sistemáticas o ensayos clínicos (aleatorizados o no)

- Probable: estudios analíticos o descripción en tres o más reportes de casos clínicos

- Posible: menos de tres casos reportados o por recomendaciones de grupos de expertos (12).
Además, para los reportes de caso que proporcionaron información suficiente, se aplicó el Roussel Uclaf Causality Assessment Method (RUCAM) (13) para determinar la causalidad del medicamento sospechoso.

\section{RESULTADOS}

Fueron identificados 531 artículos. Al retirar los duplicados, se obtuvieron 488 referencias, de las cuales 46 fueron incluidas, además de 10 artículos considerados relevantes para el tema (figura 1). Con la información de estos artículos, se identificaron 12 medicamentos (acetaminofén, alfametildopa, metotrexato, saquinavir, nevirapina, propiltiouracilo, metimazol, carbimazol, nitrofurantoína, ácido

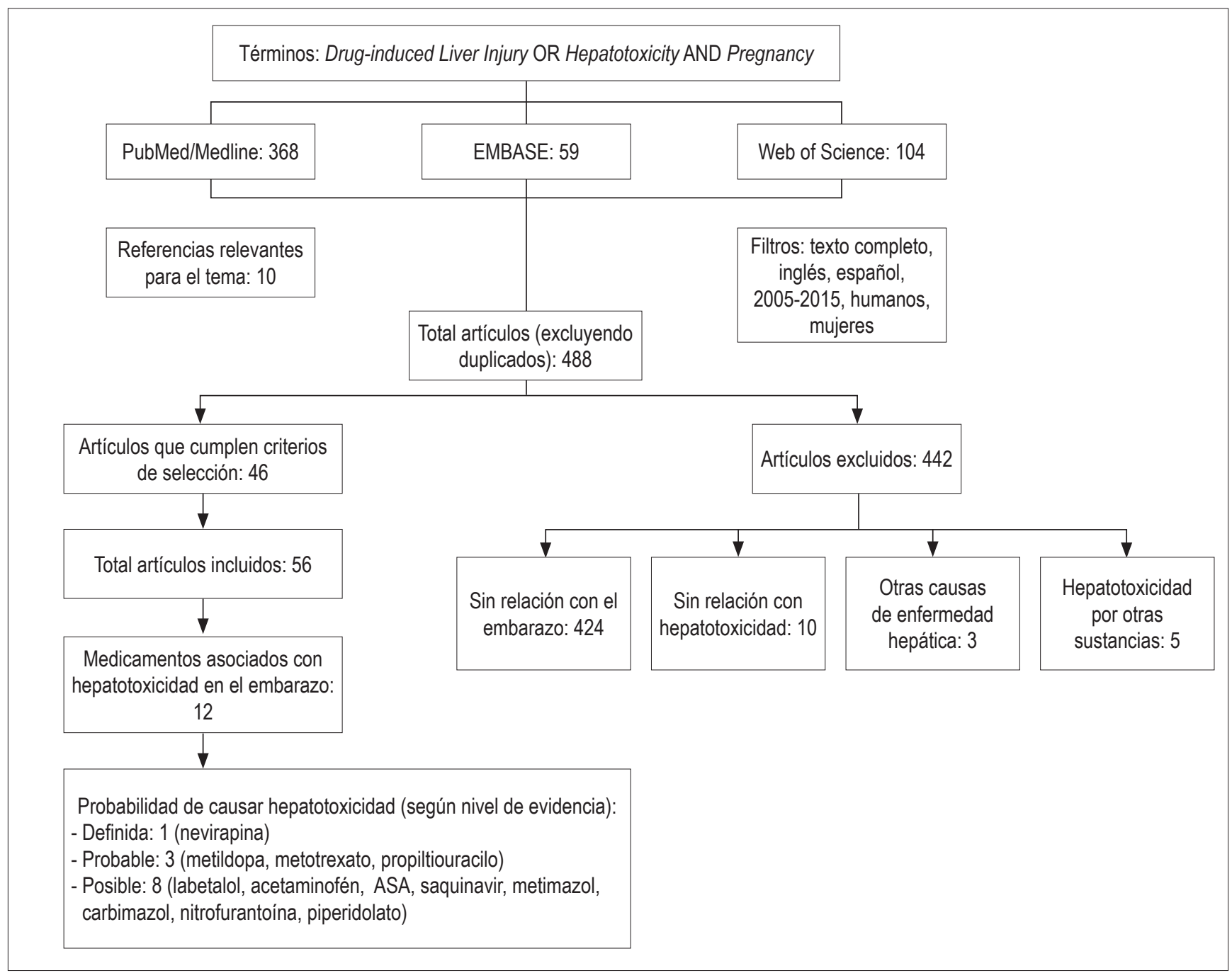

Figura 1. Resultados generales de la revisión estructurada: hepatotoxicidad en el embarazo causada por medicamentos. 
acetilsalicílico y piperidolato), con probabilidad de causar toxicidad hepática en el embarazo; además, se determinaron las principales características asociadas (tabla 1). A continuación se detallan algunos datos relevantes de los medicamentos que fueron identificados en los reportes de caso publicados y en los que se valoró la probabilidad de causar hepatotoxicidad en el embarazo.

\section{Analgésicos/antipiréticos}

Acetaminofén: usado para controlar el dolor en una dosis considerable. En una mujer de 22 años de edad se identificó toxicidad hepática a los 14 días, que requirió trasplante hepático y finalización de su embarazo con misoprostol (14). El problema de toxicidad se manifestó por dolor abdominal, ictericia, daño hepático y necrosis hepática en el feto $(14,15)$. En general, se acepta que la toxicidad se debe al metabolito del acetaminofén, la N-acetil-pbenzoquinona imina.

\section{Antihipertensivos}

Alfametildopa: se reportaron 5 casos en pacientes con toxicidad hepática, la cual se presentó entre las semanas 2 y 9, después de iniciar el tratamiento en mujeres con edad entre los 22 y 40 años. Se debió suspender el medicamento para que mejoraran la elevación de transaminasas y los síntomas, como ictericia, prurito, fatiga, anorexia, náuseas y vómitos (16).

Metildopa y labetalol: se reportó 1 caso de una mujer que usaba concomitantemente estos medicamentos para tratar la hipertensión arterial. Tuvo síntomas como ictericia y prurito a las 20 semanas de embarazo, presentó insuficiencia hepática y requirió trasplante hepático; no fue posible establecer cuál medicamento fue el responsable del daño hepático (17).

\section{Antineoplásicos}

Metotrexato: usado para el tratamiento de la enfermedad trofoblástica gestacional. Se asoció con hepatotoxicidad en 7 reportes de casos, caracterizados por aumento de las enzimas hepáticas, suspensión del medicamento y cambio a otra farmacoterapia (18).

\section{Antirretrovirales}

La farmacoterapia con medicamentos antiretrovirales incluye, en sus objetivos, prevenir la trasmisión del virus de la inmunodeficiencia humana (VIH) de madre a hijo $(7,19,20)$. La toxicidad hepática por antirretrovirales tiene una frecuencia de aparición del 5\% (21).

Saquinavir: generó 1 caso de hepatotoxicidad al mes de comenzar la terapia antirretroviral $(2000 \mathrm{mg} /$ día $)$, en una paciente con 29 semanas de embarazo, quien presentó una lesión de tipo hepatocelular, caracterizada por elevación de transaminasas y hepatitis. Se recomendó suspender el medicamento para conseguir una mejoría de los síntomas (22).

Nevirapina: la probabilidad de este medicamento para causar hepatotoxicidad se concibe como definida; sin embargo, el riesgo de toxicidad por exposición indirecta en los neonatos no ha sido definido (20). Las reacciones tóxicas pueden aparecer entre los 10 y los 280 días de iniciado el tratamiento (23-26). La hepatotoxicidad se ha presentado en pacientes con edad entre los 18 y 45 años $(21,24-27)$, con elevación de las transaminasas y rash $(23,26-37)$. Se presentó 1 caso de ductopenia, caracterizado por un tipo de lesión colestásica (25). El manejo se fundamenta en la suspensión de la nevirapina y cambio en la farmacoterapia (23, 26-29). La frecuencia de hepatotoxicidad por este medicamento es variable, entre el $0,5 \%$ y el $20 \%(24,30$, $31,34,37-39$ ).

\section{Antitiroideos}

Estos medicamentos han sido usados para tratar mujeres embarazadas con problemas de hipertiroidismo y enfermedad de Graves. La frecuencia de hepatotoxicidad para este grupo de fármacos oscila entre $0,1 \%-0,2 \%$, y es de tipo idiosincrásica (11).

Propiltiouracilo: en una mujer con 10-14 semanas de embarazo se reportó edema, ictericia, prurito y rash (4042). El manejo dado a las pacientes fue la suspensión del medicamento y el tratamiento de los síntomas con farmacoterapia (40-44). La toxicidad hepática fue de tipo idiosincrásica, con consecuencias como insuficiencia hepática, trasplante hepático, muerte materna y neonatal (42, 45-47). Aun así, en caso de ser necesario, su uso se prefiere durante el primer trimestre de embarazo, por su menor incidencia de efectos teratogénicos con respecto al metimazol $(45,47)$.

Metimazol/carbimazol: además de la posible toxicidad hepática de tipo colestásica, estos fármacos pueden causar en el feto atresia coanal y esofágica $(11,47)$, onfalocele, además de hipertiroidismo fetal (11). En general, por su elevada probabilidad de causar efectos teratogénicos, su uso debe evitarse durante el primer trimestre de embarazo (45). 
Tabla 1. Características asociadas con los medicamentos hepatotóxicos usados en el embarazo

\begin{tabular}{|c|c|c|c|c|c|c|c|c|c|c|}
\hline $\begin{array}{c}\text { Grupo } \\
\text { farmacológico }\end{array}$ & $\begin{array}{l}\text { Fármaco (cantidad } \\
\text { de artículos) - } \\
\text { (clasificación } \\
\text { del riesgo en } \\
\text { embarazo, según } \\
\text { FDA) }\end{array}$ & $\begin{array}{l}\text { Dosis (mg/ } \\
\text { día) }\end{array}$ & $\begin{array}{l}\text { Tiempo de } \\
\text { aparición del } \\
\text { evento }\end{array}$ & $\begin{array}{l}\text { Manifestaciones } \\
\text { clínicas }\end{array}$ & $\begin{array}{c}\text { Probabilidad de } \\
\text { hepatotoxicidad } \\
\text { (12) } \\
\text { (Valoración } \\
\text { RUCAM) }\end{array}$ & $\begin{array}{c}\text { Prevalencia } \\
\text { hepatotoxicidad }\end{array}$ & $\begin{array}{l}\text { Semanas } \\
\text { de } \\
\text { embarazo }\end{array}$ & $\begin{array}{l}\text { Factores de } \\
\text { riesgo }\end{array}$ & $\begin{array}{l}\text { Desenlace y } \\
\text { manejo }\end{array}$ & $\begin{array}{l}\text { Valores de } \\
\text { enzimas } \\
\text { hepáticas }\end{array}$ \\
\hline $\begin{array}{l}\text { Analgésicos / } \\
\text { antipiréticos }\end{array}$ & $\begin{array}{l}\text { Acetaminofén (2) - } \\
\text { (B) (4) }\end{array}$ & $\begin{array}{l}8000-9000 \\
(14)\end{array}$ & $\begin{array}{l}14 \text { días } \\
\text { después (14) }\end{array}$ & $\begin{array}{l}\text { Dolor abdominal, } \\
\text { ictericia escleral, } \\
\text { encefalopatía, } \\
\text { ictericia, } \\
\text { insuficiencia } \\
\text { hepática fulminante } \\
\text { (14) } \\
\text { Necrosis hepática } \\
\text { en el feto } \\
\text { En la madre: } \\
\text { anorexia, náuseas, } \\
\text { vómitos, ictericia, } \\
\text { daño hepático (15) }\end{array}$ & $\begin{array}{l}\text { Posible } \\
\text { (RUCAM: } \\
\text { probable) }\end{array}$ & Sin información & $19(14)$ & $\begin{array}{l}\text { Mujer de } 22 \\
\text { años de edad } \\
\text { (14) }\end{array}$ & $\begin{array}{l}\text { Trasplante de } \\
\text { hígado, término } \\
\text { del embarazo } \\
\text { con misoprostol } \\
(14)\end{array}$ & $\begin{array}{l}\text { ALT: } 1436 \\
\text { U/L AST: } 1064 \\
\text { U/L } \\
\text { BT: } 2,8 \mathrm{mg} / \mathrm{dL} \\
(14)\end{array}$ \\
\hline Analgésicos & $\begin{array}{l}\text { Ácido acetilsalicílico } \\
\text { (1) (categoría C/D) } \\
\text { (3) }\end{array}$ & $\begin{array}{l}\text { Sin } \\
\text { información }\end{array}$ & $\begin{array}{l}3 \text { semanas } \\
\text { después (49) }\end{array}$ & $\begin{array}{l}\text { Elevación de } \\
\text { enzimas hepáticas } \\
(49)\end{array}$ & $\begin{array}{l}\text { Posible } \\
\text { (RUCAM: } \\
\text { probable) }\end{array}$ & Sin información & $9(49)$ & $\begin{array}{l}\text { Mujer de } 36 \\
\text { años (49) }\end{array}$ & Sin información & $\begin{array}{l}\text { BT: } 215,5 \\
\text { umol/L (49) }\end{array}$ \\
\hline Antihipertensivos & $\begin{array}{l}\text { Alfametildopa (2) } \\
\text { (categoría B/C) (3) }\end{array}$ & $\begin{array}{l}500-1000 \\
(16)\end{array}$ & $\begin{array}{l}2-9 \text { semanas } \\
\text { después }(16) \\
6 \text { semanas } \\
(53)\end{array}$ & $\begin{array}{l}\text { Elevación de las } \\
\text { transaminasas, } \\
\text { hepatitis fulminante, } \\
\text { ictericia, prurito, } \\
\text { orina oscura, heces } \\
\text { pálidas, malestar, } \\
\text { fatiga, anorexia, } \\
\text { náuseas y vómitos } \\
(16,53)\end{array}$ & $\begin{array}{l}\text { Probable } \\
\text { (RUCAM: } \\
\text { probable) }\end{array}$ & Sin información & $\begin{array}{l}12-13 \\
\text { semanas } \\
\text { (mujeres } \\
\text { de } 33 \text { y } 34 \\
\text { años de } \\
\text { edad) (16, } \\
53 \text { ) }\end{array}$ & $\begin{array}{l}\text { Mujeres de } \\
22,30,33, \\
34,37 \text { y } 40 \\
\text { años de edad } \\
(16,53)\end{array}$ & $\begin{array}{l}\text { Suspensión del } \\
\text { medicamento y } \\
\text { mejora de los } \\
\text { síntomas (16) }\end{array}$ & $\begin{array}{l}\text { AST: } 412 \mathrm{U} / \mathrm{L} \\
\text { ALT: } 685 \mathrm{U} / \mathrm{L} \\
\text { BT: } 160 \mu \mathrm{mol} / \mathrm{L} \\
\text { FA: } 301 \mathrm{U} / \mathrm{L} \\
(16)\end{array}$ \\
\hline Antihipertensivos & $\begin{array}{l}\text { Uso concomitante de } \\
\text { metildopa (categoría } \\
\text { B/C) (3) y labetalol } \\
\text { (categoría B/C) } \\
\text { (3) (1) }\end{array}$ & $\begin{array}{l}\text { Sin } \\
\text { información }\end{array}$ & $\begin{array}{l}8 \text { semanas } \\
\text { (metildopa) } \\
\text { y } 4 \text { semanas } \\
\text { (labetalol) (17) }\end{array}$ & $\begin{array}{l}\text { Elevación de } \\
\text { enzimas hepáticas, } \\
\text { ictericia, prurito, } \\
\text { coluria, acolia (17) }\end{array}$ & $\begin{array}{l}\text { Posible } \\
\text { (RUCAM: } \\
\text { probable } \\
\text { para ambos } \\
\text { medicamentos) }\end{array}$ & Sin información & $20(17)$ & $\begin{array}{l}\text { Mujer de } \\
39 \text { años de } \\
\text { edad, HTA, } \\
\text { diabetes, } \\
\text { obesidad } \\
\text { (17) }\end{array}$ & $\begin{array}{l}\text { Suspensión del } \\
\text { medicamento } \\
\text { Trasplante } \\
\text { hepático (17) }\end{array}$ & $\begin{array}{l}\text { ALT: } 1406 \mathrm{U} / \mathrm{L} \\
\text { AST: } 2701 \mathrm{U} / \mathrm{L} \\
\text { FA: } 159 \mathrm{U} / \mathrm{L} \\
\text { BT:304 mmol/L } \\
(17)\end{array}$ \\
\hline Antineoplásicos & $\begin{array}{l}\text { Metotrexato (1) } \\
\text { (categoría D/X) (4) }\end{array}$ & $\begin{array}{l}40 \mathrm{mg} / \mathrm{m}^{2} \\
\text { intramuscular } \\
\text { (18) }\end{array}$ & $\begin{array}{l}\text { Sin } \\
\text { información }\end{array}$ & $\begin{array}{l}\text { Elevación de } \\
\text { enzimas hepáticas } \\
(18)\end{array}$ & $\begin{array}{l}\text { Probable } \\
\text { (RUCAM: no se } \\
\text { pudo determinar) }\end{array}$ & Sin información & $\begin{array}{l}3,2 \pm 2,9 \\
(18)\end{array}$ & $\begin{array}{l}66 \text { mujeres } \\
\text { con edad } \\
\text { promedio } \\
28,7 \pm 9,5 \\
(18)\end{array}$ & $\begin{array}{l}\text { Suspensión del } \\
\text { medicamento } \\
\text { y cambio a } \\
\text { actinomicina } \\
\text { D (18) }\end{array}$ & $\begin{array}{l}\text { Sin } \\
\text { información }\end{array}$ \\
\hline
\end{tabular}


Tabla 1. Características asociadas con los medicamentos hepatotóxicos usados en el embarazo. Continuación

\begin{tabular}{|c|c|c|c|c|c|c|c|c|c|c|}
\hline $\begin{array}{c}\text { Grupo } \\
\text { farmacológico }\end{array}$ & $\begin{array}{c}\text { Fármaco (cantidad } \\
\text { de artículos) - } \\
\text { (clasificación } \\
\text { del riesgo en } \\
\text { embarazo, según } \\
\text { FDA) }\end{array}$ & $\begin{array}{l}\text { Dosis (mg/ } \\
\text { día) }\end{array}$ & $\begin{array}{l}\text { Tiempo de } \\
\text { aparición del } \\
\text { evento }\end{array}$ & $\begin{array}{l}\text { Manifestaciones } \\
\text { clínicas }\end{array}$ & $\begin{array}{c}\text { Probabilidad de } \\
\text { hepatotoxicidad } \\
\text { (12) } \\
\text { (Valoración } \\
\text { RUCAM) }\end{array}$ & $\begin{array}{c}\text { Prevalencia } \\
\text { hepatotoxicidad }\end{array}$ & $\begin{array}{l}\text { Semanas } \\
\text { de } \\
\text { embarazo }\end{array}$ & $\begin{array}{l}\text { Factores de } \\
\text { riesgo }\end{array}$ & $\begin{array}{l}\text { Desenlace y } \\
\text { manejo }\end{array}$ & $\begin{array}{l}\text { Valores de } \\
\text { enzimas } \\
\text { hepáticas }\end{array}$ \\
\hline \multirow[t]{2}{*}{ Antirretrovirales } & $\begin{array}{l}\text { Nevirapina (22) } \\
\text { (categoría C) }(4, \\
6,52)\end{array}$ & $\begin{array}{l}200-400(3 \\
23,24,31 \\
32,34,54)\end{array}$ & $\begin{array}{l}\text { Desde } 10 \\
\text { días después } \\
(23-26,29 \\
31-34,55)\end{array}$ & $\begin{array}{l}\text { Elevación de } \\
\text { transaminasas } \\
(32-35,54), \text { rash } \\
(21,32-34,56) \text {, } \\
\text { hepatitis, prurito, } \\
\text { dolor abdominal } \\
\text { (24), eosinofilia } \\
\text { (34), encefalopatía } \\
\text { (7) } \\
\text { Ictericia }(24,54)\end{array}$ & $\begin{array}{l}\text { Definida } \\
\text { (RUCAM: } \\
\text { probable) (25) }\end{array}$ & $\begin{array}{l}0,5 \%(30), 1 \% \\
(56), 4 \%-6 \%(24), \\
6,8 \%(38), 13,3 \% \\
(36), 20,3 \%(31)\end{array}$ & $\begin{array}{l}20-34(24- \\
27,30-34)\end{array}$ & $\begin{array}{l}\text { Mujer entre } \\
18-45 \text { años } \\
\text { de edad } \\
\text { Virus } \\
\text { hepatitis B y } \\
\text { C }(21,54)\end{array}$ & $\begin{array}{l}\text { Suspensión del } \\
\text { medicamento } \\
(25-28) \text { y } \\
\text { manejo de } \\
\text { rash con } \\
\text { betametasona } \\
\text { (34) } \\
\text { Muerte (7) }\end{array}$ & $\begin{array}{l}\text { ALT: } 1005(7), \\
235,295,241 \\
\text { (34), 179 (25) } \\
\text { AST: } 1276(7), \\
\text { 309, 152, 306 } \\
\text { (34), 103 (25) } \\
\text { FA: } 496 \mathrm{U} / \mathrm{L} \\
\text { BT: } 10,5 \mathrm{mg} / \\
\text { dL (25) }\end{array}$ \\
\hline & $\begin{array}{l}\text { Saquinavir (1) } \\
\text { (categoría B) }(6,52)\end{array}$ & $2000(22)$ & $\begin{array}{l}1 \text { mes } \\
\text { después (22) }\end{array}$ & $\begin{array}{l}\text { Hepatitis cistolítica, } \\
\text { elevación de } \\
\text { transaminasas (22) }\end{array}$ & $\begin{array}{l}\text { Posible } \\
\text { (RUCAM: no se } \\
\text { pudo determinar) }\end{array}$ & $7 \%(22)$ & $29,3(22)$ & $\begin{array}{l}\text { Sin } \\
\text { información }\end{array}$ & $\begin{array}{l}\text { Suspensión del } \\
\text { medicamento } \\
\text { y resolución de } \\
\text { síntomas (22) }\end{array}$ & $\begin{array}{l}\text { AST: } 303 \\
\text { ALT: } 351 \text { (22) }\end{array}$ \\
\hline \multirow[t]{2}{*}{ Antitiroideos } & $\begin{array}{l}\text { Metimazol (3) / } \\
\text { Carbimazol (2) } \\
\text { (categoría D) (4) }\end{array}$ & $\begin{array}{l}\text { Carbimazol: } \\
10-30(11) \\
\text { Metimazol: } \\
\text { sin } \\
\text { información }\end{array}$ & $\begin{array}{l}17 \text { días } \\
\text { después }(40, \\
45)\end{array}$ & $\begin{array}{l}\text { Defectos } \\
\text { congénitos: atresia } \\
\text { coanal y esofágica } \\
(11,47), \text { onfalocele } \\
\text { e hipertiroidismo } \\
\text { fetal (11) }\end{array}$ & $\begin{array}{l}\text { Posible } \\
\text { (RUCAM: no se } \\
\text { pudo determinar) }\end{array}$ & $0,1 \%-0,2 \%(11)$ & $\begin{array}{l}\text { Sin } \\
\text { información }\end{array}$ & $\begin{array}{l}\text { Sin } \\
\text { información }\end{array}$ & $\begin{array}{l}\text { Suspensión del } \\
\text { medicamento } \\
\text { (11) }\end{array}$ & $\begin{array}{l}\text { Sin } \\
\text { información }\end{array}$ \\
\hline & $\begin{array}{l}\text { Propiltiouracilo (8) } \\
\text { (categoría D) (4) }\end{array}$ & $\begin{array}{l}50-1400 \\
(40-44,46)\end{array}$ & $\begin{array}{l}17 \text { días } \\
\text { después }(40, \\
45)\end{array}$ & $\begin{array}{l}\text { Elevación de } \\
\text { transaminasas, } \\
\text { ictericia }(41-44) \text { e } \\
\text { hipertiroidismo fetal } \\
\text { (11), prurito, rash } \\
\text { (40), insuficiencia } \\
\text { hepática }(45,46)\end{array}$ & $\begin{array}{l}\text { Probable } \\
\text { (RUCAM: } \\
\text { probable) (41, } \\
42)\end{array}$ & $\begin{array}{l}0,1 \%-0,2 \%(11 \\
43) \\
3,9 \%(44)\end{array}$ & $\begin{array}{l}10-25(41- \\
42,46)\end{array}$ & $\begin{array}{l}\text { Edades de } \\
22 \text { y } 36 \text { años } \\
\text { de edad } \\
(41-44,46)\end{array}$ & $\begin{array}{l}\text { Trasplante de } \\
\text { hígado (45-47), } \\
\text { muerte fetal } \\
(45) \\
\text { Suspensión del } \\
\text { medicamento } \\
(32,41-44)\end{array}$ & $\begin{array}{l}\text { ALT: } 399 \mathrm{IU} / \mathrm{L} \\
\text { AST: } 590 \mathrm{IU} / \mathrm{L} \\
\text { FA: } 677 \mathrm{IU} / \mathrm{L} \\
\text { BT: } 15,9 \mathrm{mg} / \\
\mathrm{dL}(41)\end{array}$ \\
\hline Antibióticos & $\begin{array}{l}\text { Nitrofurantoína (1) } \\
\text { (categoría B) }(4,6)\end{array}$ & $100(48)$ & $\begin{array}{l}10 \text { dias } \\
\text { después (48) }\end{array}$ & $\begin{array}{l}\text { Elevación de las } \\
\text { transaminasas (48) }\end{array}$ & $\begin{array}{l}\text { Posible } \\
\text { (RUCAM: } \\
\text { probable) }\end{array}$ & Sin información & $36(48)$ & $\begin{array}{l}\text { Mujer de } 31 \\
\text { años de edad } \\
(48)\end{array}$ & $\begin{array}{l}\text { Evolución } \\
\text { favorable (48) }\end{array}$ & $\begin{array}{l}\text { AST: } 420 \mathrm{U} / \mathrm{L} \\
\text { ALT: } 861 \mathrm{U} / \mathrm{L} \\
(48)\end{array}$ \\
\hline Anticolinérgicos & $\begin{array}{l}\text { Piperidolato (1) } \\
\text { (categoría C) }\end{array}$ & $\begin{array}{l}\text { Sin } \\
\text { información }\end{array}$ & $\begin{array}{l}7 \text { semanas } \\
\text { después }(49)\end{array}$ & $\begin{array}{l}\text { Elevación de } \\
\text { enzimas hepática } \\
(49)\end{array}$ & $\begin{array}{l}\text { Posible } \\
\text { (RUCAM: } \\
\text { posible) }\end{array}$ & Sin información & $13(49)$ & $\begin{array}{l}\text { Mujer de } 36 \\
\text { años (49) }\end{array}$ & Sin información & $\begin{array}{l}\text { BT: } 389,9 \\
\text { umol/L (49) }\end{array}$ \\
\hline
\end{tabular}

ALT: alanino-aminotransferasa; AST: aspartato-aminotransferasa; BT: bilirrubina total; FA: fosfatasa alcalina; HTA: hipertensión arterial. 


\section{Antibióticos}

Nitrofurantoína: se dio el caso de una paciente con 36 semanas de embarazo quien presentó toxicidad hepática por nitrofurantoína indicada para una infección del tracto urinario. La única manifestación presentada fue la elevación de las transaminasas. Luego de suspender el medicamento, tuvo una evolución favorable (48).

\section{Otros medicamentos}

Ácido acetilsalicílico y piperidolato: se presentaron dos casos de mujeres de 36 años de edad con daño hepático por estos medicamentos, que mostraron elevación de las enzimas hepáticas a las 9 semanas de embarazo para ácido acetilsalicílico y 13 semanas, para piperidolato (49).

\section{DISCUSIÓN}

El presente trabajo identificó 12 medicamentos, de 7 grupos farmacológicos, con probabilidad de causar toxicidad hepática en el embarazo: acetaminofén y ácido acetilsalicilico (analgésicos), alfametildopa y labetalol (antihipertensivos), metotrexato (antineoplásicos), saquinavir y nevirapina (antirretrovirales), propiltiouracilo, metimazol y carbimazol (antitiroideos), nitrofurantoina (antibióticos) y piperidolato (anticolinérgicos). En este sentido, aunque han sido reportados problemas de toxicidad hepática por medicamentos psiquiátricos (9), esta revisión estructurada no encontró evidencia de una relación causal clara entre el uso de estos medicamentos y hepatotoxicidad en el embarazo, lo que podría deberse a la información limitada sobre este tema.

En general, el establecimiento de causalidad de hepatotoxicidad en el embarazo requiere de discernir entre la causa medicamentosa y otras patologías propias del embarazo, como es el caso de la colestasis intrahepática en el tercer trimestre, asociada con cambios hormonales y polimorfismos (50-56).

La tipificación de los medicamentos, al igual que la caracterización de los principales factores influyentes en este problema de salud, podría contribuir a una identificación temprana de problemas relacionados con estos agentes farmacológicos. En este sentido, las mujeres que se encuentran entre la segunda y tercera década de edad podrían tener un mayor riesgo de presentar problemas en el hígado, debido al uso de medicamentos como el metotrexato, la alfametildopa y el propiltiouracilo $(16,18,40,46)$. Las dosis usadas por las pacientes que presentaron casos de hepatotoxicidad en el embarazo estuvieron dentro de las habituales, excepto para el caso del acetaminofén (8-9 g/día) (14), la cual fue, erróneamente, considerada como segura por la paciente.
Para medicamentos como el acetaminofén, el ácido acetilsalicílico, el piperidolato, la nitrofurantoína, el metotrexato y la alfametildopa, la información sobre la frecuencia de aparición de las reacciones tóxicas en el hígado durante la gestación es escasa, mientras que para los fármacos antitiroideos, la frecuencia de aparición se puede encontrar entre $0,1 \%-0,2 \%$ de la población embarazada que usa estos medicamentos (11). El tiempo de aparición de las reacciones tóxicas después de administrado el medicamento para los antitiroideos (40) o el acetaminofén (14) se estimó en días, mientras que para medicamentos como el saquinavir (22), la alfametildopa (16) o el piperidolato (49), el orden fue de meses. Estas reacciones hepatotóxicas aparecieron cuando las pacientes tenían alrededor de la tercera semana de embarazo en el caso del metotrexato (18), y cuando las pacientes se encontraban en la décima o más semanas de embarazo para acetaminofén, alfametildopa, nitrofurantoína, nevirapina, saquinavir y antitiroideos (16, $22,33,46,48)$.

Según la clasificación de riesgo de la FDA, los antitiroideos (D) (4) y el metotrexato (D/X) (4) son los medicamentos con mayor riesgo de causar daño en el feto e inclusive la muerte (45); sin embargo, en esta revisión, con el acetaminofén (clasificado en la categoría B) (4), se presentó 1 caso de hepatotoxicidad en una mujer embarazada, cuyo desenlace fue el trasplante hepático materno y la muerte fetal (14).

La elevación de las transaminasas es una manifestación frecuente e importante del daño hepático que causan los medicamentos cuando se usan en el embarazo $(11,16$, $18,22,32-35)$. En este contexto, en el caso del acetaminofén, la nitrofurantoína, la alfametildopa y la nevirapina, el aumento en los valores de la aspartato-aminotransferasa (AST) y alanino-aminotranferasa (ALT) fue mayor de 10 veces el límite superior normal $(7,14,16,48)$. Además, otros síntomas inespecíficos, como la ictericia, los vómitos, el prurito y la anorexia, pueden aparecer en estas pacientes $(15,16,34,40)$. Por su parte, en el feto, el acetaminofén puede causar necrosis hepática (15), mientras que el metimazol y carbimazol pueden causar defectos congénitos (11, 47). El manejo que se dio en los casos de hepatotoxicidad en mujeres embarazadas fue la suspensión del medicamento sospechoso, con lo cual se logró, en algunos casos, mejorar la sintomatología.

Relacionado con la nevirapina, se podría decir que hay dudas sobre su seguridad en el embarazo, tal como se documenta con la variada incidencia reportada en los estudios $(30,31,36)$. En este sentido, es importante destacar que, aunque el uso de nevirapina durante el embarazo parece carecer de efectos teratogénicos (8), puede causar toxicidad hepática en la gestante $(7,34)$. 
En cuanto a la probabilidad de causar hepatotoxicidad de los medicamentos identificados en esta revisión, la mayoría de ellos presentó diferencias en las valoraciones (según el nivel de evidencia y la escala RUCAM), debido a los dos diferentes métodos usados para determinarla.

Acorde con esta revisión, en el caso de mujeres en embarazo y que utilizaron medicamentos con posible toxicidad hepática, además de la dosis y tiempo de exposición al fármaco, la edad de la gestante y el tiempo de gestación deberían ser variables objeto de valoración y seguimiento clínico. Esta práctica podría contribuir a controlar el riesgo de desenlaces fatales para la madre o el feto asociados con hepatotoxicidad; sin embargo, esta medida requiere de trabajos orientados a explorar dicho efecto. En este sentido, es importante resaltar que el riesgo de hepatotoxicidad, por el grupo de medicamentos identificados, oscila entre definida, probable y posible; información que podría ser utilizada para mejorar el análisis del riesgo/beneficio.

\section{CONCLUSIÓN}

El acetaminofén, la alfametildopa, el metotrexato, el saquinavir, la nevirapina, el propiltiouracilo, el metimazol, el carbimazol, la nitrofurantoína, el ácido acetilsalicílico y el piperidolato podrían causar hepatotoxicidad en pacientes embarazadas. Además de la dosis y del tiempo de exposición al fármaco, la edad y el tiempo de gestación podrían influir en la presentación y gravedad de la hepatotoxicidad. La información disponible sobre toxicidad hepática por medicamentos en el embarazo es limitada.

\section{Financiación}

El grupo Promoción y Prevención Farmacéutica recibió financiación de la convocatoria de sostenibilidad 2014-2015 del Comité para el Desarrollo de la Investigación (CODI) de la Universidad de Antioquia, Medellín, Colombia.

\section{REFERENCIAS}

1. Vázquez-Benitez E. El uso de algunos farmacos y sus riesgos durante el embarazo. Gac Méd Mex. 1996;132(5):541-3.

2. Lorente $S$, Serrano T. Enfermedades hepáticas propias del embarazo. Rev Esp Enf Dig. 2010;102(8):505-6.

3. Gallego M, Delgado L, Campos M, et al. Actualización del uso de fármacos durante el embarazo: categorías de riesgo. Farm Hosp. 2014;38(4):364-78.

4. Abad F, Pons J, Micó M, et al. Categorías de riesgo de los medicamentos utilizados durante el embarazo: Guía rápida de consulta. Farm Aten Prim. 2005;3(2):49-61.

5. Pérez A, Allende M, Agustín M, et al. Teratogénesis: clasificaciones. Farm Hosp. 2002;26(3):171-7.
6. Vallano A, Arnau J. Antimicrobianos y embarazo. Enferm Infecc Microbiol Clin. 2009;27(9):536-42.

7. Lyons F, Hopkins S, Kelleher B, et al. Maternal hepatotoxicity with nevirapine as part of combination antiretroviral therapy in pregnancy. HIV Med. 2006;7(4):255-60.

8. Coster L, Kumar P. Contemporary role of nevirapine in HIV treatment. AIDS Rev. 2012;14(2):132-44.

9. Gómez E, Lizbeth C. Manejo del trastorno bipolar en el embarazo. Rev Med MD. 2012;3(3):154-62.

10. Petit D. The use of antithyroid drugs. Calif Med. 1951;74(2):99-104.

11. Cassina M, Doná M, Di Gianantonio E, et al. Pharmacologic treatment of hyperthyroidism during pregnancy. Birth Defects Res Clin Mol Teratol. 2012;94(8):612-9.

12. Amariles P, Giraldo N, Faus M. Interacciones medicamentosas: aproximación para establecer y evaluar su relevancia clínica. Med Clin (Barc). 2007;129(1):27-35.

13. Danan G, Benichou C. Causality assessment of adverse reactions to drugs-I. A novel method based on the conclusions of international consensus meetings: application to drug-induced liver injuries. J Clin Epidemiol. 1993;46(11):1323-30.

14. Thornton S, Minns A. Unintentional chronic acetaminophen poisoning during pregnancy resulting in liver transplantation. J Med Toxicol. 2012;8:176-8.

15. Wilkes J, Clark L, Herrera J. Acetaminophen overdose in pregnancy. South Med J. 2005;98(11):1118-23.

16. Slim R, Ben Salem C, Hmouda H, et al. Hepatotoxicity of alpha-methyldopa in pregnancy. J Clin Pharm Ther. 2010;35:361-3.

17. Firoz T, Webber D, Rowe H. Drug-induced fulminant hepatic failure in pregnancy. Obstet Med. 2015;8(4):190-2.

18. Gilani M, Fariba B, Behtash N, et al. The WHO score predicts treatment outcome in low risk gestational trophoblastic neoplasia patients treated with weekly intramuscular methotrexate. J Cancer Res Ther. 2013;9(1):38-43.

19. Solís I, Muñoz E, Tomás J, et al. Características maternas en una cohorte de gestantes con infección por el VIH-1. Med Clin (Barc). 2006;127(4):121-5.

20. Minniear T, Zeh C, Polle N, et al. Rash, Hepatotoxicity and hyperbilirubinemia among Kenyan infants born to hivinfected women receiving triple-antiretroviral drugs for the prevention of mother-to-child HIV transmission. Pediatr Infect Dis J. 2012;31(11):1155-7.

21. Snijdewind I, Smit C, Godfried M, et al. HCV coinfection, an important risk factor for hepatotoxicity in pregnant women starting antiretroviral therapy. J Infect. 2012;64(4):409-16.

22. Brunet $\mathrm{C}$, Reliquet $\mathrm{V}$, Jovelin $\mathrm{T}$, et al. Effectiveness and safety of saquinavir / ritonavir in HIV-infected pregnant women: INEMA cohort. Med Mal Infect. 2012;42:421-8.

23. Bottaro E, Huberman M, Iannella M, et al. Nevirapine-associated toxicity in clinical practice in Buenos Aires, Argentina. J Int Assoc Physicians AIDS Care. 2010;9(5):306-12.

24. Peters P, Polle N, Zeh C, et al. Nevirapine-associated hepatotoxicity and rash among HIV-infected pregnant women in Kenya. J Int Assoc Physicians AIDS Care. 2012;11(2):142-9. 
25. Kochar R, Nevah M, Lukens F, et al. Vanishing bile duct syndrome in human immunodeficiency virus : Nevirapine hepatotoxicity revisited. World J Gastroenterol. 2010;16(26):3335-8.

26. Jamisse L, Balkus J, Hitti J, et al. Antiretroviral-associated toxicity among HIV-1 - Seropositive pregnant women in Mozambique receiving nevirapine-based regimens. Acquir Immune Defic Syndr. 2007;44(4):371-6.

27. Timmermans S, Tempelman C, Godfried M, et al. Nelfinavir and nevirapine side effects during pregnancy. AIDS Rev. 2005; 19:795-9.

28. Phanuphak N, Apornpong T, Teeratakulpisarn S, et al. Nevirapine-associated toxicity in HIV-infected Thai men and women, including pregnant women. HIV Med. 2007;8:357-66.

29. Coffie P, Tonwe-Gold B, Tanon A, et al. Incidence and risk factors of severe adverse events with nevirapine-based antiretroviral therapy in HIV-infected women. MTCT-Plus program, Abidjan, Côte d'Ivoire. BMC Infect Dis. 2010;10:188.

30. Kilewo C, Karlsson K, Ngarina M, et al. Prevention of mother-to-child transmission of HIV-1 through breastfeeding by treating mothers with triple antiretroviral therapy in Dar es Salaam, Tanzania: The Mitra Plus study. J Acquir Immune Defic Synd. 2009;52(3):406-16.

31. Kondo W, Carraro E, Prandel E, et al. Nevirapine-induced side effects in pregnant women - experience of a Brazilian university hospital. BJID. 2007;11(6):544-8.

32. Natarajan U, Pym A, Mcdonald C, et al. Safety of nevirapine in pregnancy. HIV Medicine. 2007;8:64-9.

33. Marazzi M, Germano P, Liotta G, et al. Safety of nevirapinecontaining antiretroviral triple therapy regimens to prevent vertical transmission in an African cohort of HIV-1-infected pregnant women. HIV Medicine. 2006;7:338-44.

34. Joy S, Poi M, Hughes L, et al. Third-trimester maternal toxicity with nevirapine use in pregnancy. Obstet Gynecol. 2005;106(5):1032-8.

35. Ouyang D, Shapiro D, Lu M, et al. Increased risk of hepatotoxicity in HIV-infected pregnant women receiving antiretroviral therapy independent of nevirapine exposure. AIDS Rev. 2009;23(18):2425-30.

36. Ouyang D, Brogly S, Lu M, et al. Lack of increased hepatotoxicity in $\mathrm{HIV}$-infected pregnant women receiving nevirapine compared to other antiretrovirals. AIDS Rev. 2010;24(1):109-14.

37. Pillay P, Black V. Safety, strength and simplicity of efavirenz in pregnancy. South Afr J HIV Med. 2012;13(1):28-33.

38. Ford N, Calmy A, Andrieux-Meyer I, et al. Adverse events associated with nevirapine use in pregnancy: a systematic review and meta-analysis. AIDS Rev. 2013;27:1135-43.

39. Aaron E, Kempf M-C, Criniti S, et al. Adverse events in a cohort of HIV infected pregnant and non-pregnant women treated with nevirapine versus non-nevirapine antiretroviral medication. PLoS One. 2010;5(9):e12617.

40. Ekiz B, Soysal N, Kirkizlar O, et al. Effectiveness of preoperative plasmapheresis in a pregnancy complicated by hyper- thyroidism and anti-thyroid drug-associated angioedema. Gynecol Endocrinol. 2013;29(5):508-10.

41. Miyamura T, Kanda T, Minemura S, et al. Acute liver failure associated with propylthiouracil in a pregnant 26-year-old woman. Case Rep Gastroenterol. 2013;7(2):240-4.

42. Sequeira E, Wanyonyi S, Dodia R. Severe propylthiouracilinduced hepatotoxicity in pregnancy managed successfully by liver transplantation: A case report. J Med Case Rep. 2011;5:461.

43. Lo J, Rivkees S, Chandra $M$, et al. Gestational thyrotoxicosis, antithyroid drug use and neonatal outcomes within an integrated healthcare delivery system. Thyroid. 2015;25(6):698-705.

44. Yoshihara A, Noh J, Watanabe N, et al. Frequency of adverse events of antithyroid drugs administered during pregnancy. J Thyroid Res. 2014;2014:952352.

45. Akmal A, Kung J. Propylthiouracil, and methimazole, and carbimazole-related hepatotoxicity. Expert Opin Drug Saf. 2014;13(10):1397-406.

46. Glinoer D, Cooper D. The propylthiouracil dilemma. 2012;19(5):402-7.

47. Azizi F, Amouzegar A. Management of hyperthyroidism during pregnancy and lactation. Eur J Endocrinol. 2011;164:871-6.

48. Aksamija A, Horvat G, Habek D, et al. Nitrofurantoininduced acute liver damage in pregnancy. Arh Hig Rada Toksikol. 2009;60:357-61.

49. Tsuyoshi H, Nishijima K, Takahashi J, et al. Management of druginduced hyperbilirubinaemia in early pregnancy. S Afr J Obstet Gynaecol. 2013;19(1):22-3.

50. Dietrich C, Geier A. Effect of drug transporter pharmacogenetics on cholestasis. Expert Opin Drug Metab Toxicol. 2014;10(11):1533-51.

51. Magnus P, Meier P, Stieger B. Genetic determinants of druginduced cholestasis and intrahepatic cholestasis of pregnancy. Semin Liver Dis. 2010;30(2):147-59.

52. Peña J, Ramos J, Pedrol P, et al. Tratamiento de la mujer embarazada infectada por el VIH. Enferm Infecc Microbiol Clin. 2002;20(Suppl 2):29-34.

53. Ali T, Srinivasan N, Le V, et al. Alpha-methyldopa hepatotoxicity in pregnancy. J Coll Physicians Surg Pak. 2009; 19(2):125-6.

54. Andreotti M, Pirillo M, Liotta G, et al. The impact of HBV or $\mathrm{HCV}$ infection in a cohort of HIV-infected pregnant women receiving a nevirapine-based antiretroviral regimen in Malawi. BMC Infect Dis. 2014;14:180.

55. Bera E, Mia R. Safety of nevirapine in HIV-infected pregnant women initiating antiretroviral therapy at higher CD4 counts: A systematic review and meta-analysis. S Afr Med. 2012;102(11):855-9.

56. Cecchini $D$, Urueña A, Trinidad $P$, et al. HIV and pregnancy: maternal and neonatal evolution. Medicina (B Aires). $2011 ; 71(5): 432-6$. 